\title{
Developing Attractor Analysis Techniques for a Compositional Boolean Network Framework
}

\author{
Hanin Abdulrahman \\ School of Computing, Newcastle University, \\ Newcastle upon Tyne, UK \\ Faculty of Computer and Information Sciences, \\ Princess Nourah Bint Abdulrahman University, \\ Riyadh, Saudi Arabia \\ H.Y.I.Abdulrahman2@ncl.ac.uk
}

\author{
Jason Steggles \\ School of Computing, Newcastle University, \\ Newcastle upon Tyne, UK \\ Jason.Steggles@ncl.ac.uk
}

\begin{abstract}
Boolean networks are an important qualitative modelling technique that provide techniques for analysing the attractors (important cyclic behaviour) in a model. However, their practical application is limited by the state space explosion problem and this had led to researchers considering compositional techniques. In this paper we take a recently developed compositional framework for Boolean networks based on using logical connectives to merge entities and extend it with compositional techniques for attractor analysis. Our approach is based on using strongly connected components to identify potential cyclic behaviour taking into account the interference arising from a composition. We develop tool support for our approach and illustrate its practical application by a case study.

Index Terms-Boolean network, composition, attractor analysis, qualitative modelling
\end{abstract}

\section{INTRODUCTION}

Boolean networks [11], [12] are a qualitative modelling approach based on using Boolean states to model regulatory entities. They have been shown to be an important tool for modelling and analysing biological regulatory networks [8], [14], [20] and in particular, using attractor analysis (i.e. identifying key cyclic behaviour) can provide crucial insights into biological systems [10], [22].

In practice, the application of Boolean network techniques is limited by the well-known state space explosion problem where the exponential growth of the state space severely limits the size of the model. This limitation has led to the development of compositional techniques to support Boolean networks (for example, see [3], [16], [30]). In recent work [3], [4] an interesting new compositional approach was proposed based on composing two Boolean networks by merging entities using Boolean operators such as conjunction. This compositional framework set out to provide a foundation for both engineering Boolean networks and decomposing them to aid analysis. The initial work so far has focused on the preservation of the behaviour of subnetworks in a composition [3]. However, no support has so far been developed for compositionally identifying the attractors in a composed model. Given the

We gratefully acknowledge the support provided by the Faculty of Computer and Information Sciences, Princess Nourah Bint Abdulrahman University. importance of attractor analysis [10], [22] this is a crucial area that needs addressing to support the practical application of this compositional framework.

In this paper we present the first initial work on developing techniques and tools for compositionally identifying attractors in the given compositional framework [3], [4]. The approach we develop is based on analysing each subnetwork's interference state graph [3], a state graph that extends a Boolean network normal behaviour with the additional dynamics arising from interference in a composition. We use the strongly connected components (SCCs) [15] in the interference state graphs to identify cyclic behaviour which is then selectively merged based on a new property called interference alignment. We formally show our approach is correct by proving that it is sound and complete for attractor identification.

We use the techniques we develop to formulate an algorithm for compositional attractor identification which we use to develop tool support. The practical application of the developed techniques and tools is illustrated by applying them to a case study based on a regulatory network for cell differentiation [21], [23] found in the bacteria Caulobacter crescentus [13].

Given the importance of attractor analysis a range of related work on the compositional analysis of attractors exists in the literature. In [16], [28]-[30] a range of approaches are considered for compositional attractor analysis based on decomposing a Boolean network into blocks (subnetworks with special input entities duplicated from other blocks). The decomposition is optimised by using the SCCs of a Boolean network's interaction graph. Similar approaches are developed in [5], [9] where the underlying attractor identification technique is based on a SAT-based approach. The approach presented here is significantly different to the above work since it is based on a general framework for composition that allows models to be engineered from subparts. In particular, it uses logical operators to merge entities and focuses on the behavioural interference this can generate.

This paper is organised as follows. In Section 2 we briefly introduce Boolean networks and the existing compositional framework. In Section 3 we extend the existing compositional framework by developing new techniques for compositional 
identifying attractors. In Section 4 we consider developing tool support and then illustrate our approach using a case study. Finally, in Section 6 we make some concluding remarks.

\section{BOOLEAN NETWORKS AND COMPOSITION}

A Boolean network [1], [12] is a qualitative model that consists of a set of regulatory entities which have a binary state, where 1 represents that an entity is active and 0 represents that an entity is inactive. The behaviour of each entity is regulated by other entities in the model and the set of these entities is referred to as the neighbourhood of an entity.

We can define a Boolean network formally as follows.

Definition 1. A Boolean Network $\mathcal{B N}$ is a tuple $\mathcal{B N}=$ $(G, N, F)$ where:

i) $G=\left\{g_{1}, \ldots, g_{n}\right\}$ is a non-empty, finite set of entities; ii) $N=\left(N\left(g_{1}\right), \ldots, N\left(g_{n}\right)\right)$ is a tuple of neighbourhoods, such that $N\left(g_{i}\right) \subseteq G$ is the neighbourhood of $g_{i}$; and iii) $F=\left(F\left(g_{1}\right), \ldots, F\left(g_{n}\right)\right)$ is a tuple of next-state functions, where $F\left(g_{i}\right): \mathbb{B}^{\left|N\left(g_{i}\right)\right|} \rightarrow \mathbb{B}$ defines the next state of $g_{i}$.

Let $\mathcal{B N}=(G, N, F)$ be a Boolean network with entities $G=\left\{g_{1}, \ldots, g_{n}\right\}$, for some $n \geq 1$. Then a global state for $\mathcal{B N}$ is a tuple $\left(s_{1}, \ldots, s_{n}\right)$, where $s_{i}$ represents the state of entity $g_{i}$. We let $S_{\mathcal{B N}}=\mathbb{B}^{|G|}$ be the set of all possible global states for $\mathcal{B N}$.

The global state of a Boolean network can be updated either synchronously [27] (all entities update their state simultaneously) or asynchronously [7] (entities update their state independently). In this paper we focus on the widely used synchronous update semantics [24] and let $S_{1} \stackrel{\mathcal{B N}}{\longrightarrow} S_{2}$ represent a (synchronous) update step where $S_{2}$ is the global state resulting from simultaneously updating the state of each entity $g_{i}$ by applying its update function $F\left(g_{i}\right)$ to the appropriate neighbourhood of states in $S_{1}$.
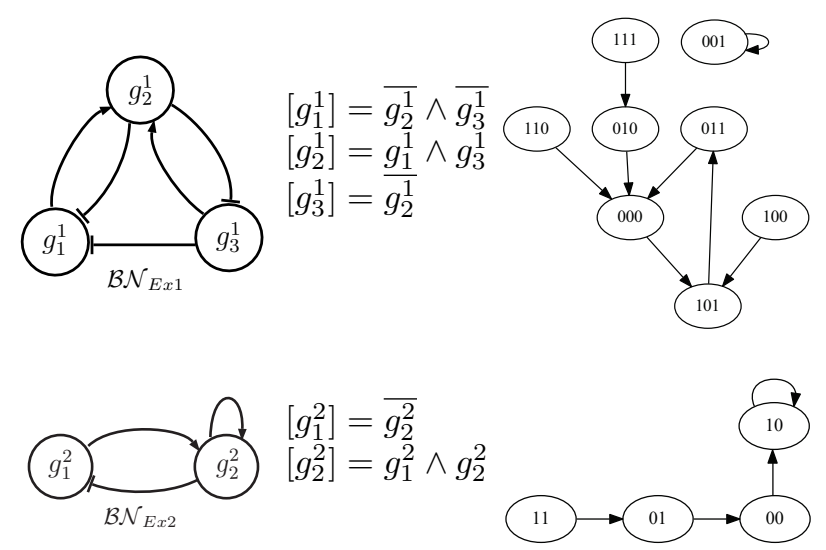

Fig. 1: The interaction graph, next-state functions and state graphs for example Boolean Networks $\mathcal{B N}_{E x 1}$ and $\mathcal{B N}_{E x 2}$

As an illustrative example, consider the Boolean networks $\mathcal{B N}_{E x 1}$ and $\mathcal{B N}_{E x 2}$ presented in Figure 1. Given the global state 101 in $\mathcal{B N}_{E x 1}$ (denoting that entity $g_{1}^{1}=1, g_{2}^{1}=0$, and $g_{3}^{1}=1$ ) we can apply the next-state functions to make a synchronous update step $101 \stackrel{\mathcal{B N}_{E x 1}}{\longrightarrow} 011$.
The complete synchronous behaviour of a Boolean network $\mathcal{B N}$ can be concisely represented as a state graph $S G(\mathcal{B N})=$ $\left(S_{\mathcal{B N}}, \stackrel{\mathcal{B N}}{\longrightarrow}\right)$. Examples of state graphs are given in Figure 1 for $\mathcal{B N}_{E x 1}$ and $\mathcal{B N}_{E x 2}$. A path (often referred to as a trace) for a Boolean network is an infinite sequence of global states

$$
\left\langle S_{0}, S_{1}, S_{2}, \ldots\right\rangle
$$

such that $S_{i} \in S_{\mathcal{B N}}$ and $S_{i} \stackrel{\mathcal{B N}}{\longrightarrow} S_{i+1}$, for $i \in \mathbb{N}$. We let $\operatorname{Path}(S G(\mathcal{B N}))$ represent the set of all such (infinite) paths. Since the global state space for a Boolean network is finite all paths must enter an attractor cycle [12], [26]. For example, the Boolean network $\mathcal{B N}_{E x 1}$ (see Figure 1) has two attractors: $[001,001]$ and $[101,011,000,101]$. Identifying attractors is crucial when analysing a Boolean network as they represent key biologically relevant behaviour [10], [22].

A compositional framework for Boolean networks was developed by Alkhudhayr and Steggles [2]-[4] based on composing Boolean networks by merging entities using Boolean connectives. In the sequel, let $\mathcal{B N}_{1}=\left(G_{1}, N_{1}, F_{1}\right)$ and $\mathcal{B N}_{2}=\left(G_{2}, N_{2}, F_{2}\right)$ be two Boolean networks with entities $G_{1}=\left\{g_{1}^{1}, \ldots, g_{n}^{1}\right\}$ and $G_{2}=\left\{g_{1}^{2}, \ldots, g_{m}^{2}\right\}$. To compose $\mathcal{B N}_{1}$ and $\mathcal{B N}_{2}$ we select an entity from each, say $g_{1}^{1}$ and $g_{1}^{2}$ respectively, to merge into a new entity $g^{c}$. The nextstate function for $g^{c}$ is defined by using conjunction (or any idempotent Boolean connective) to combine the next-state functions $F_{1}\left(g_{1}^{1}\right)$ and $F_{2}\left(g_{1}^{2}\right)$. The resulting composed model is denoted by $\mathcal{C}\left(\mathcal{B N}_{1}, \mathcal{B N}_{2}, g_{1}^{1}, g_{1}^{2}\right)$.

To illustrate the idea, consider composing the Boolean networks $\mathcal{B N}_{E x 1}$ and $\mathcal{B N}_{E x 2}$ (Figure 1) by merging entities $g_{1}^{1}$ and $g_{1}^{2}$ using conjunction. The result is a new Boolean network $\mathcal{C}\left(\mathcal{B N}_{E x 1}, \mathcal{B N}_{E x 2}, g_{1}^{1}, g_{1}^{2}\right)$ (see Figure 2) in which the entities $g_{1}^{1}$ and $g_{1}^{2}$ have been replaced by a single composed entity $g^{c}$ whose next state function is defined by

$$
\left[g^{c}\right]=\left(\overline{g_{2}^{1}} \wedge \overline{g_{3}^{1}}\right) \wedge \overline{g_{2}^{2}}
$$

In the sequel, we let a global state $S \in \mathcal{S}_{\mathcal{C}}$ in the composed model $\mathcal{C}=\mathcal{C}\left(\mathcal{B N}_{1}, \mathcal{B N}_{2}, g_{1}^{1}, g_{1}^{2}\right)$ have the form $S=\left(\begin{array}{llllllll}s & s_{2}^{1} & \ldots & s_{n}^{1} & s_{2}^{2} & \ldots & s_{m}^{2}\end{array}\right) \in \mathcal{S}_{\mathcal{C}}$, where $s$ is the state of the new merged entity $g^{c}$ and $s_{j}^{i}$ is the state of entity $g_{j}^{i}$. We define projection operators to allow the global states for the subnetworks to be retrieved:

$$
\mathcal{P}_{\mathcal{B N}_{1}}(S)=\left(\begin{array}{llll}
s & s_{2}^{1} & \ldots & s_{n}^{1}
\end{array}\right), \quad \mathcal{P}_{\mathcal{B N}_{2}}(S)=\left(\begin{array}{llll}
s & s_{2}^{2} & \ldots & s_{m}^{2}
\end{array}\right)
$$

The projection operators can be lifted to a path $\alpha \in$ $\operatorname{Path}(S G(\mathcal{C}))$ and a set of paths in the standard way.

The following definition (taken from [3]) defines how to merge global states and paths.

Definition 2. Let $S^{1}=\left(\begin{array}{lll}s_{1}^{1} & \ldots & s_{n}^{1}\end{array}\right) \in \mathcal{S}_{\mathcal{B N}_{1}}$ and $S^{2}=$ $\left(s_{1}^{2} \ldots s_{m}^{2}\right) \in \mathcal{S}_{\mathcal{B N}_{2}}$, and $\mathcal{C}=\mathcal{C}\left(\mathcal{B N}_{1}, \mathcal{B N}_{2}, g_{1}^{1}, g_{1}^{2}\right)$. We define $S^{1} \wedge_{g_{1}^{1}, g_{1}^{2}} S^{2} \in \mathcal{S}_{\mathcal{C}}$ by merging the state of $g_{1}^{1}$ with $g_{1}^{2}$

$$
S^{1} \wedge_{g_{1}^{1}, g_{1}^{2}} S^{2}=\left(\left(s_{1}^{1} \wedge s_{1}^{2}\right) s_{2}^{1} \ldots s_{n}^{1} s_{2}^{2} \ldots s_{m}^{2}\right)
$$

For any paths $\alpha_{1}=\left\langle S_{1}^{1}, S_{2}^{1}, \ldots\right\rangle \in \operatorname{Path}\left(S G\left(\mathcal{B N}_{1}\right)\right)$ and $\alpha_{2}=\left\langle S_{1}^{2}, S_{2}^{2}, \ldots\right\rangle \in \operatorname{Path}\left(S G\left(\mathcal{B N}_{2}\right)\right)$ we define

$$
\alpha_{1} \wedge_{g_{1}^{1}, g_{1}^{2}} \alpha_{2}=\left\langle S_{1}^{1} \wedge_{g_{1}^{1}, g_{1}^{2}} S_{1}^{2}, S_{2}^{1} \wedge_{g_{1}^{1}, g_{1}^{2}} S_{2}^{2}, \ldots\right\rangle
$$



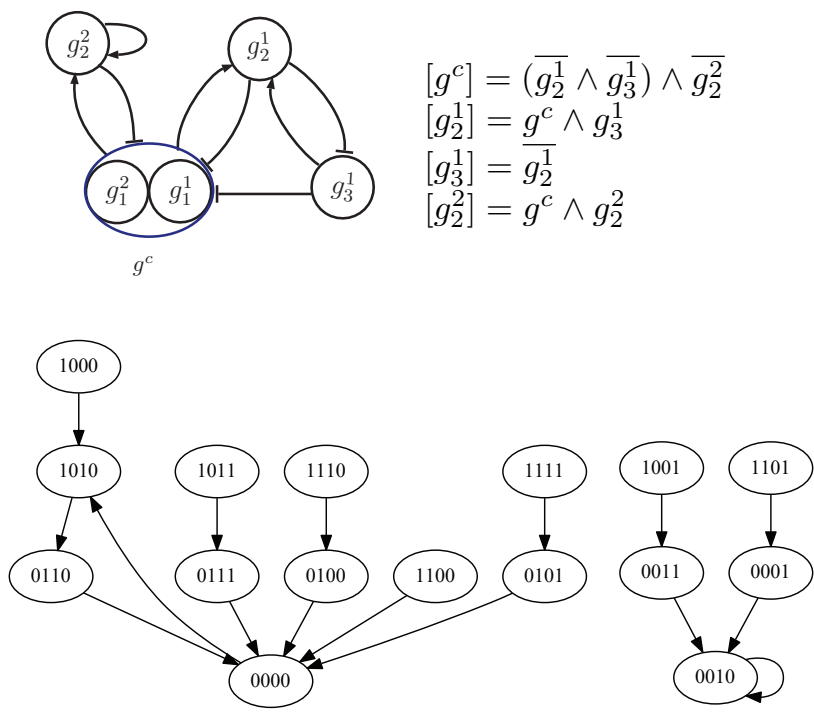

Fig. 2: Composed model $\mathcal{C}\left(\mathcal{B N}_{E x 1}, \mathcal{B N}_{E x 2}, g_{1}^{1}, g_{1}^{2}\right)$ resulting from composing $\mathcal{B N}_{E x 1}$ with $\mathcal{B N}_{E x 2}$ on $g_{1}^{1}$ and $g_{1}^{2}$ (using conjunction)

In a slight abuse of notation, we normally use $S^{1} \wedge S^{2}$ and $\alpha_{1} \wedge \alpha_{2}$ to represent $S^{1} \wedge_{g_{1}^{1}, g_{1}^{2}} S^{2}$ and $\alpha_{1} \wedge_{g_{1}^{1}, g_{1}^{2}} \alpha_{2}$ respectively.

The results and tools developed for this compositional framework [3] have so far focused on the preservation of a subnetwork's behaviour in a composition. One important concept is that two subnetworks used in a composition can interfere with each other to produce new behaviour in the composed model.

For example, consider $\mathcal{C}\left(\mathcal{B N}_{E x 1}, \mathcal{B N}_{E x 2}, g_{1}^{1}, g_{1}^{2}\right)$ (see Figure 2) and suppose the composed model is in global state 1001. Then the underlying Boolean networks will want to make the following transitions: $100 \stackrel{\mathcal{B N}_{E x 1}}{\longrightarrow} 101$ and $11 \stackrel{\mathcal{B N}_{E x 2}}{\longrightarrow} 01$. In other words, entity $g_{1}^{1}$ in $\mathcal{B N}_{E x 1}$ would like to transition from 1 to 1 but entity $g_{1}^{2}$ in $\mathcal{B N}_{E x 2}$ would like to transition from 1 to 0 . The merged entity $g^{c}$ will therefore transition to $0 \wedge 1=0$ resulting in the next state 0011 ; this means the underlying behaviour of $\mathcal{B N}_{E x 1}$ has been interfered with. So interference in a composition based on conjunction occurs whenever a merged entity wants to transition to 1 but its merged counterpart wants to transition to 0 .

In order to formalise the possible interference that can occur between composed Boolean networks the concept of a state graph is extended to an interference state graph [3] which has additional edges representing the behaviour that could result from interference. The idea (based on using conjunction) is that whenever the entity to be merged transitions to 1 then we add another edge to the state graph to represent that the transition could instead go to 0 due to interference.

Definition 3. We define the interference state graph $S G_{g_{i}}(\mathcal{B N})$ for $\mathcal{B N}$ on $g_{i} \in G$ by $S G_{g_{i}}(\mathcal{B N})=\left(\mathcal{S}_{\mathcal{B N}}, \underset{g_{i}}{\stackrel{\mathcal{N}}{\longrightarrow}}\right)$,
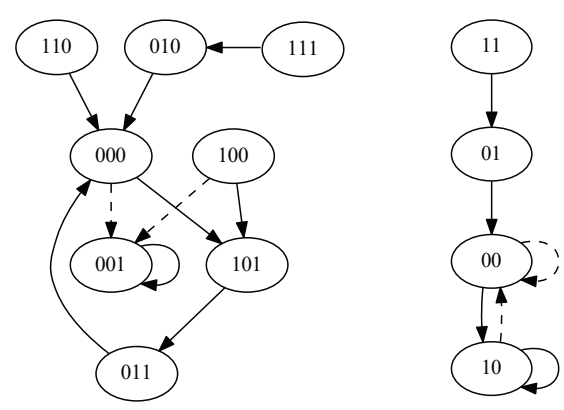

$$
S G_{g_{1}^{1}}\left(\mathcal{B N}_{E x 1}\right) \quad S G_{g_{1}^{2}}\left(\mathcal{B N}_{E x 2}\right)
$$

Fig. 3: The interference state graphs for $\mathcal{B N}_{E x 1}$ on $g_{1}^{1}$ and $\mathcal{B N}_{E x 2}$ on $g_{1}^{2}$

where

$$
\begin{aligned}
\stackrel{\mathcal{B N}}{\longrightarrow}= & \stackrel{\mathcal{B N}}{\longrightarrow} \cup\left\{\left(\left(\begin{array}{lllllllll}
s_{1} & \ldots & s_{n}
\end{array}\right),\left(\begin{array}{lllllll}
s_{1}^{\prime} & \ldots & s_{i-1}^{\prime} & 0 & s_{i+1}^{\prime} & \ldots & s_{n}^{\prime}
\end{array}\right)\right)\right. \\
& \left.\left(\begin{array}{lllllll}
s_{1} & \ldots & s_{n}
\end{array}\right) \stackrel{\mathcal{B N}}{\longrightarrow}\left(\begin{array}{lllllll}
s_{1}^{\prime} & \ldots & s_{i-1}^{\prime} & 1 & s_{i+1}^{\prime} & \ldots & s_{n}^{\prime}
\end{array}\right)\right\}
\end{aligned}
$$

For example, consider the interference state graphs for $\mathcal{B N}_{E x 1}$ and $\mathcal{B N}_{E x 2}$ presented in Figure 3.

The interference state graph captures all the potential behaviour a Boolean network can have under composition as the following result from [3] shows.

Theorem 1. Given a composition $\mathcal{C}=\mathcal{C}\left(\mathcal{B N}_{1}, \mathcal{B N}_{2}, g_{1}^{1}, g_{1}^{2}\right)$ we have:

i) $\mathcal{P}_{\mathcal{B N}_{1}}(\operatorname{Path}(\mathcal{C})) \subseteq \operatorname{Path}\left(S G_{g_{1}^{1}}\left(\mathcal{B N}_{1}\right)\right)$; and

ii) $\mathcal{P}_{\mathcal{B N}_{2}}(\operatorname{Path}(\mathcal{C})) \subseteq \operatorname{Path}\left(S G_{g_{1}^{2}}\left(\mathcal{B N}_{2}\right)\right)$.

\section{COMPOSITIONALly IDENTIFying ATtRACTORS}

In this section we develop a new novel technique for compositionally identifying the attractors in a composed model by finding cyclic behaviour in each subnetwork's interference state graph that can be merged to produce an attractor.

Recall that a strongly connected component (SCC) [15] is a maximal set of vertices in a graph such that any two vertices are mutually reachable. We define formally what we mean by an SCC for an interference state graph as follows.

Definition 4. Let $S G_{g}(\mathcal{B N})=\left(S_{\mathcal{B N}}, \frac{\mathcal{B N}}{g}\right)$ be an interference state graph for $\mathcal{B N}$ and let $\varphi=\left(\mathcal{S}_{\varphi}, \stackrel{\varphi}{\rightarrow}\right)$ be a non-empty subgraph of $S G_{g}(\mathcal{B N})$ (i. e. $\mathcal{S}_{\varphi} \subseteq S_{\mathcal{B N}}$ is a non-empty set of global states and $\stackrel{\varphi}{\rightarrow} \underset{g}{\stackrel{\mathcal{B N}}{\rightarrow}}$ is a non-empty edge relation). Then $\varphi$ is a Strongly Connected Component $(S C C)$ for $S G_{g}(\mathcal{B N})$ iff the following holds: i) for any two states $S, T \in \mathcal{S}_{\varphi}$ there is a directed path from $S$ to $T$; and ii) $\varphi$ is maximal (adding any further nodes or edges from $S G_{g}(\mathcal{B N})$ to $\varphi$ breaks the above connectivity property).

We let $S C C\left(S G_{g}(\mathcal{B N})\right)$ denote the set of all SCCs for an interference state graph $S G_{g}(\mathcal{B N})$. 
As an example, consider the SCCs depicted in Figure 4 for the interference state graphs $S G_{g_{1}^{1}}\left(\mathcal{B N}_{E x 1}\right)$ and $S G_{g_{1}^{2}}\left(\mathcal{B N} \mathcal{N}_{E x 2}\right)$.

We use the SCCs in a subnetwork's interference state graph to identify cyclic behaviour that can be merged to generate attractors in the composed model. Let $\varphi \in S C C\left(S G_{g}(\mathcal{B N})\right)$ and let $\alpha$ be an infinite path over $\varphi$. We say that $\alpha$ is a cyclic path iff there exists $k \in \mathbb{N}$ and $S_{1}, \ldots, S_{k} \in \mathcal{S}_{\varphi}$ such that

$$
\alpha=\left\langle S_{1}, \ldots, S_{k}, S_{1}, \ldots, S_{k}, S_{1}, \ldots, S_{k}, \ldots\right\rangle
$$

We let $\operatorname{CPaths}(\varphi)$ denote the set of all cyclic paths for an $\operatorname{SCC} \varphi$.

We merge the cyclic paths generated from the SCCs in the subnetworks to identify attractors using a new property called interference alignment to check when cyclic paths are compatible.
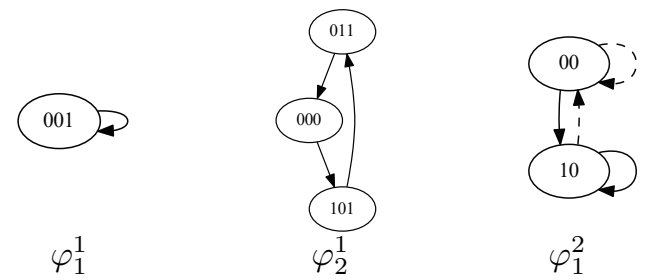

Fig. 4: The SCCs $\varphi_{1}^{1}$ and $\varphi_{2}^{1}$ in $S G_{g_{1}^{1}}\left(\mathcal{B N}_{E x 1}\right)$ and $\varphi_{1}^{2}$ in $S G_{g_{1}^{2}}\left(\mathcal{B N}_{E x 2}\right)$

Given two states $S_{1}, S_{2} \in S_{\mathcal{B N}}$, we refer to a state transition step $S_{1} \stackrel{\mathcal{B N}}{\longrightarrow} S_{2}$ as a normal step iff $S_{1} \stackrel{\mathcal{B N}}{\longrightarrow} S_{2}$ (i.e. it does not require interference). A step $S_{1} \underset{g}{\stackrel{\mathcal{B N}}{\longrightarrow}} S_{2}$ is referred to as an interference step iff it is not a normal step (i.e. it does require interference). For a Boolean network $\mathcal{B N}$ We define the entity state projection $\mathcal{P}_{g_{i}}(S)=s_{i}$, for any entity $g_{i}$ in $\mathcal{B N}$ and $S=\left(s_{1}, \ldots, s_{n}\right) \in S_{\mathcal{B N}}$. The projection operators can be lifted to a path $\alpha \in \operatorname{Path}(S G(\mathcal{B N}))$ and a set of paths in the standard way.

Definition 5. Let $\alpha_{1}=\left\langle S_{1}, S_{2}, \ldots\right\rangle \in \operatorname{Path}\left(S G_{g_{1}^{1}}\left(\mathcal{B N}_{1}\right)\right)$ and $\alpha_{2}=\left\langle T_{1}, T_{2}, \ldots\right\rangle \in \operatorname{Path}\left(S G_{g_{1}^{2}}\left(\mathcal{B N}_{2}\right)\right)$. Then we say that $\alpha_{1}$ and $\alpha_{2}$ interference align iff $\mathcal{P}_{g_{1}^{1}}\left(\alpha_{1}\right)=\mathcal{P}_{g_{1}^{2}}\left(\alpha_{2}\right)$ and for any $i \in \mathbb{N}$, we have $S_{i} \underset{g_{1}^{1}}{\stackrel{\mathcal{B N}_{1}}{\longrightarrow}} S_{i+1}$ and $T_{i} \underset{g_{1}^{2}}{\stackrel{\mathcal{B N}_{2}}{\longrightarrow}} T_{i+1}$ are not both interference steps.

Interference alignment captures when paths can be merged to create a path in the composed model by checking to ensure that interference actually occurs at the points required in each path. This is formally shown by the following result.

Lemma 2. Let $\alpha_{1} \in \operatorname{Path}\left(S G_{g_{1}^{1}}\left(\mathcal{B N}_{1}\right)\right)$ and $\alpha_{2} \in$ $\operatorname{Path}\left(S G_{g_{1}^{2}}\left(\mathcal{B N}_{2}\right)\right)$. Then if $\alpha_{1}$ and $\alpha_{2}$ interference align then

$$
\alpha_{1} \wedge \alpha_{2} \in \operatorname{Path}\left(S G\left(\mathcal{C}\left(\mathcal{B N}_{1}, \mathcal{B N}_{2}, g_{1}^{1}, g_{1}^{2}\right)\right)\right)
$$

Proof. Let $\alpha_{1}=\left\langle S_{1}, S_{2}, \ldots\right\rangle \in \operatorname{Path}\left(S G_{g_{1}^{1}}\left(\mathcal{B N}_{1}\right)\right)$ and $\alpha_{2}=\left\langle T_{1}, T_{2}, \ldots\right\rangle \in \operatorname{Path}\left(S G_{g_{1}^{2}}\left(\mathcal{B N}_{2}\right)\right)$ such that $\alpha_{1}$ and $\alpha_{2}$ interference align. Let $\mathcal{C}=\mathcal{C}\left(\mathcal{B N}_{1}, \mathcal{B N}_{2}, g_{1}^{1}, g_{1}^{2}\right)$. To show $\alpha_{1} \wedge \alpha_{2} \in \operatorname{Path}(S G(\mathcal{C}))$ it suffices to show that for any $i \in \mathbb{N}$

$$
S_{i} \wedge T_{i} \stackrel{\mathcal{C}}{\rightarrow} S_{i+1} \wedge T_{i+1}
$$

By the definition of interference alignment we know that $\mathcal{P}_{g_{1}^{1}}\left(S_{i}\right)=\mathcal{P}_{g_{1}^{2}}\left(T_{i}\right)$ and so by idempotency of conjunction we have

$$
\mathcal{P}_{g_{1}^{1}}\left(S_{i}\right) \wedge \mathcal{P}_{g_{1}^{2}}\left(T_{i}\right)=\mathcal{P}_{g_{1}^{1}}\left(S_{i}\right)=\mathcal{P}_{g_{1}^{2}}\left(T_{i}\right)
$$

By assumption we have

$$
\text { (Step 1) } S_{i} \underset{g_{1}^{1}}{\stackrel{\mathcal{B N}_{1}}{\longrightarrow}} S_{i+1} \text { and (Step 2) } T_{i} \underset{g_{1}^{2}}{\stackrel{\mathcal{B N}_{2}}{\longrightarrow}} T_{i+1}
$$

and at least one of these steps must be normal. If both of these steps are normal then (1) follows straightforwardly by interference alignment and definition of $\mathcal{C}$.

Alternatively, suppose Step 1 is an interference step then Step 2 must be normal. It follows that

$$
\mathcal{P}_{g_{1}^{1}}\left(S_{i+1}\right)=\mathcal{P}_{g_{1}^{2}}\left(T_{i+1}\right)=0
$$

and that $\mathcal{B N}_{1}$ intended for $g_{1}^{1}$ to transition to state 1 . Then the result follows since

$$
\mathcal{P}_{g_{1}^{1}}\left(S_{i+1}\right) \wedge \mathcal{P}_{g_{1}^{2}}\left(T_{i+1}\right)=1 \wedge \mathcal{P}_{g_{1}^{2}}\left(T_{i+1}\right)
$$

A similar argument applies if Step 2 is assumed to be the interference step.

We now have a basis for compositionally identifying attractors. Suppose we have two SCCs, $\varphi_{1} \in \operatorname{SCC}\left(S G_{g_{1}^{1}}\left(\mathcal{B N}_{1}\right)\right)$ and $\varphi_{2} \in S C C\left(S G_{g_{1}^{2}}\left(\mathcal{B N}_{2}\right)\right)$, and cyclic paths $\alpha_{1} \in$ $\operatorname{CPaths}\left(\varphi_{1}\right)$ and $\alpha_{2} \in \operatorname{CPaths}\left(\varphi_{2}\right)$. Then if these cyclic paths interference align then they can be merged $\alpha_{1} \wedge \alpha_{2}$ producing a path that must represent an attractor in the composed model $\mathcal{C}\left(\mathcal{B N}_{1}, \mathcal{B N}_{2}, g_{1}, g_{2}\right)$.

To illustrate this idea consider the SCCs $\varphi_{1}^{1}$ and $\varphi_{2}^{2}$ for $S G_{g_{1}^{1}}\left(\mathcal{B N} \mathcal{N}_{E x 1}\right)$ and $S G_{g_{1}^{2}}\left(\mathcal{B N} \mathcal{N}_{E x 2}\right)$ (see Figure 4) and the cyclic paths $\langle 011,000,101,011,000,101,011, \ldots\rangle$ in $\varphi_{2}^{1}$ and $\langle 00,00,10,00,00,10,00, \ldots\rangle$ in $\varphi_{1}^{2}$. These two cyclic paths can be seen to interference align and so by Lemma 2 we know that the path that results from merging them

$$
\langle 0110,0000,1010,0110,0000,1010,0110 \ldots\rangle
$$

is in the composed model. It therefore follows that $[0110,0000,1010,0110]$ must be an attractor for the composed model $\mathcal{C}\left(\mathcal{B N}_{E x 1}, \mathcal{B N}_{E x 2}, g_{1}^{1}, g_{1}^{2}\right)$.

We formally prove our approach for compositionally identifying attractors is correct by showing it is sound (any attractor found is valid) and complete (all valid attractors are found).

Theorem 3. (Soundness) Let $\varphi_{1} \in S C C\left(S G_{g_{1}^{1}}\left(\mathcal{B N}_{1}\right)\right)$ and $\varphi_{2} \in S C C\left(S G_{g_{1}^{2}}\left(\mathcal{B N}_{2}\right)\right)$ be SCCs. Let $\alpha_{1} \in \operatorname{CPaths}\left(\varphi_{1}\right)$ and $\alpha_{2} \in C$ Paths $\left(\varphi_{2}\right)$ be cyclic paths such that $\alpha_{1}$ and $\alpha_{2}$ interference align. Then $\alpha_{1} \wedge \alpha_{2}$ represents an attractor in the composed model $\mathcal{C}\left(\mathcal{B N}_{1}, \mathcal{B N}_{2}, g_{1}^{1}, g_{1}^{2}\right)$.

Proof. Let $\varphi_{1} \in \operatorname{SCC}\left(S G_{g_{1}^{1}}\left(\mathcal{B N}_{1}\right)\right)$ and $\varphi_{2} \in$ $\operatorname{SCC}\left(S G_{g_{1}^{2}}\left(\mathcal{B N}_{2}\right)\right)$ be SCCs. Let $\alpha_{1} \in \operatorname{CPaths}\left(\varphi_{1}\right)$ 
and $\alpha_{2} \in$ CPaths $\left(\varphi_{2}\right)$ be cyclic paths such that $\alpha_{1}$ and $\alpha_{2}$ interference align. By above assumptions and Lemma 2 it follows

$$
\alpha_{1} \wedge \alpha_{2} \in \operatorname{Path}\left(S G\left(\mathcal{C}\left(\mathcal{B N}_{1}, \mathcal{B N}_{2}, g_{1}^{1}, g_{1}^{2}\right)\right)\right)
$$

Since $\alpha_{1}$ and $\alpha_{2}$ are cyclic paths it follows that $\alpha_{1} \wedge \alpha_{2}=$ $\left\langle S T_{1}, \ldots, S T_{k}, S T_{1}, \ldots, S T_{k}, \ldots\right\rangle$, for some $k \in \mathbb{N}$, must be a cyclic path and so $\left[S T_{1}, \ldots, S T_{k}, S T_{1}\right]$ must be an attractor in $\mathcal{C}\left(\mathcal{B N}_{1}, \mathcal{B N}_{2}, g_{1}^{1}, g_{1}^{2}\right)$.

We now consider showing the proposed approach is complete and begin with some necessary preliminary results about projecting and merging paths in a composed model.

Lemma 4. Let $\beta \in \operatorname{Path}\left(S G\left(\mathcal{C}\left(\mathcal{B N}_{1}, \mathcal{B N}_{2}, g_{1}^{1}, g_{1}^{2}\right)\right)\right)$. Then

$$
\mathcal{P}_{\mathcal{B N}_{1}}(\beta) \wedge \mathcal{P}_{\mathcal{B N}_{2}}(\beta)=\beta
$$

Proof. Let $\mathcal{C}=\mathcal{C}\left(\mathcal{B N}_{1}, \mathcal{B N}_{2}, g_{1}^{1}, g_{1}^{2}\right)$ and $\beta=$ $\left\langle S T_{1}, S T_{2}, \ldots\right\rangle \in \operatorname{Path}(S G(\mathcal{C}))$. It suffices to show that for any $i \in \mathbb{N}$ we have

$$
S T_{i}=\mathcal{P}_{\mathcal{B N}_{1}}\left(S T_{i}\right) \wedge \mathcal{P}_{\mathcal{B N}_{2}}\left(S T_{i}\right)
$$

This follows by the idempotency of conjunction and since

$$
\mathcal{P}_{g_{1}^{1}}\left(\mathcal{P}_{\mathcal{B N}_{1}}\left(S T_{i}\right)\right)=\mathcal{P}_{g_{1}^{2}}\left(\mathcal{P}_{\mathcal{B N}_{2}}\left(S T_{i}\right)\right)=\mathcal{P}_{g^{c}}\left(S T_{i}\right)
$$

Lemma 5. Let $\beta \in \operatorname{Path}\left(S G\left(\mathcal{C}\left(\mathcal{B N}_{1}, \mathcal{B N}_{2}, g_{1}^{1}, g_{1}^{2}\right)\right)\right)$. Then we must have:

i) $\mathcal{P}_{\mathcal{B N}_{1}}(\beta) \in \operatorname{Path}\left(S G_{g_{1}^{1}}\left(\mathcal{B N}_{1}\right)\right)$

ii) $\mathcal{P}_{\mathcal{B N}_{2}}(\beta) \in \operatorname{Path}\left(S G_{g_{1}^{2}}\left(\mathcal{B N}_{2}\right)\right)$

Proof. Let $\mathcal{C}=\mathcal{C}\left(\mathcal{B N}_{1}, \mathcal{B N}_{2}, g_{1}^{1}, g_{1}^{2}\right)$ and $\beta=$ $\left\langle S T_{1}, S T_{2}, \ldots\right\rangle \in \operatorname{Path}(S G(\mathcal{C}))$. To show (i) it suffices to show that for any $i \in \mathbb{N}$ there exists a step

$$
\mathcal{P}_{\mathcal{B N}_{1}}\left(S T_{i}\right) \underset{g_{1}^{1}}{\stackrel{\mathcal{B N}_{1}}{\longrightarrow}} \mathcal{P}_{\mathcal{B N}_{1}}\left(S T_{i+1}\right)
$$

If $\mathcal{P}_{\mathcal{B N}_{1}}\left(S T_{i}\right) \stackrel{\mathcal{B N}_{1}}{\longrightarrow} \mathcal{P}_{\mathcal{B N}_{1}}\left(S T_{i+1}\right)$ holds then clearly (1) holds. Otherwise, if it does not hold then by definition of $\mathcal{C}$ this must mean that the value of $g_{1}^{1}$ has been interfered with. In other words, $g_{1}^{1}$ was supposed to have next state 1 but the next state for $g_{1}^{2}$ was 0 giving a next state for $g^{c}$ of $1 \wedge 0=0$. In this case, by the definition of the interference state graph $S G_{g_{1}^{1}}\left(\mathcal{B N}_{1}\right)$ we must have (1) holds.

The proof of ii) follows along similar lines.

We can now prove the proposed compositional attractor identification approach is complete.

Theorem 6. (Completeness) Let $\alpha$ be an attractor for $\mathcal{C}\left(\mathcal{B N}_{1}, \mathcal{B N}_{2}, g_{1}^{1}, g_{1}^{2}\right)$. Then there must exist $\varphi_{1} \in$ $S C C\left(S G_{g_{1}^{1}}\left(\mathcal{B N}_{1}\right)\right)$ and $\varphi_{2} \in S C C\left(S G_{g_{1}^{2}}\left(\mathcal{B N}_{2}\right)\right)$, and cyclic paths $\alpha_{1} \in$ CPaths $\left(\varphi_{1}\right)$ and $\alpha_{2} \in$ CPaths $\left(\varphi_{2}\right)$ such that $\alpha_{1}$ and $\alpha_{2}$ interference align, and $\alpha_{1} \wedge \alpha_{2}$ results in the attractor $\alpha$.
Proof. Let $\alpha=\left[S T_{1}, S T_{2}, \ldots S T_{k}, S T_{1}\right]$ be an arbitrary attractor in the composed model $\mathcal{C}\left(\mathcal{B N}_{1}, \mathcal{B N}_{2}, g_{1}^{1}, g_{1}^{2}\right)$. Viewing $\alpha$ as an infinite cyclic path, let $\alpha_{1}=\mathcal{P}_{\mathcal{B N}_{1}}(\alpha)$ and $\alpha_{2}=\mathcal{P}_{\mathcal{B N}_{2}}(\alpha)$. By Lemma 4 it follows that $\alpha_{1} \wedge \alpha_{2}$ must result in $\alpha$. Clearly, $\alpha_{1}$ and $\alpha_{2}$ are cyclic paths since $\alpha$ is. It follows by Lemma 5 that $\alpha_{1} \in \operatorname{Path}\left(S G_{g_{1}^{1}}\left(\mathcal{B N}_{1}\right)\right)$ and $\alpha_{2} \in \operatorname{Path}\left(S G_{g_{1}^{2}}\left(\mathcal{B N}_{2}\right)\right)$. To see that $\alpha_{1}$ and $\alpha_{2}$ interference align first note that they must align $\mathcal{P}_{g_{1}^{1}}\left(\alpha_{1}\right)=\mathcal{P}_{g_{1}^{2}}\left(\alpha_{2}\right)$ by definition. It follows that the corresponding state transition steps in $\alpha_{1}$ and $\alpha_{2}$ cannot both be interference steps since they are derived from $\alpha$. Finally, since $\alpha_{1}$ and $\alpha_{2}$ are cyclic paths it can be seen that they must result from an SCC structure in their corresponding interference state graphs as required.

\section{Developing Tool Support}

In this section we use the theoretical approach outlined in the previous section as the basis to develop tool support for compositionally identifying attractors in a composed model. We begin by formulating an algorithm for identifying and merging cyclic paths from given SCCs for the subnetworks.

Let $S_{1}, S_{2} \in S_{\mathcal{B N}_{1}}$ and $S_{1} \underset{g_{1}^{1}}{\stackrel{\mathcal{B N}_{1}}{\longrightarrow}} S_{2}$, and $T_{1}, T_{2} \in S_{\mathcal{B N}_{2}}$ and $T_{1} \underset{g_{1}^{2}}{\stackrel{\mathcal{B N}_{2}}{\longrightarrow}} T_{2}$. Then we say $\left(S_{2}, T_{2}\right)$ is an interference aligned next state pair for $\left(S_{1}, T_{1}\right)$, iff $\mathcal{P}_{g_{1}^{1}}\left(S_{2}\right)=\mathcal{P}_{g_{1}^{2}}\left(T_{2}\right)$ and at least one of the state transitions is a normal step. Note for any state pair $\left(S_{1}, T_{1}\right)$ it can be shown that there is at most one interference aligned next state pair.

The pseudocode given below outlines an algorithm idAtts which given two SCCs $\varphi_{1} \in S C C\left(S G_{g_{1}^{1}}\left(\mathcal{B N}_{1}\right)\right)$ and $\varphi_{2} \in$ $S C C\left(S G_{g_{1}^{2}}\left(\mathcal{B N}_{2}\right)\right)$ finds all the attractors associated with these cyclic structures. The algorithm makes use of the following auxiliary functions:

- $\operatorname{alignSet}\left(\varphi_{1}, \varphi_{2}\right)$ returns the set of all aligned state pairs for the given SCCs and is defined by

$$
\begin{gathered}
\operatorname{align} \operatorname{Set}\left(\varphi_{1}, \varphi_{2}\right)= \\
\left\{(S, T) \mid S \in \mathcal{S}_{\varphi_{1}}, T \in \mathcal{S}_{\varphi_{2}}, \mathcal{P}_{g_{1}^{1}}(S)=\mathcal{P}_{g_{1}^{2}}(T)\right\} ;
\end{gathered}
$$

- $\operatorname{noStep}\left((S, T), \varphi_{1}, \varphi_{2}\right)$ is a Boolean function that checks if a state pair $(S, T)$ does not have an interference aligned next state pair with respect to $\varphi_{1}$ and $\varphi_{2}$;

- doStep $\left((S, T), \varphi_{1}, \varphi_{2}\right)$ returns, if it exists, the interference aligned next state pair for $(S, T)$ with respect to $\varphi_{1}$ and $\varphi_{2}$;

- $\operatorname{ext} C P($ list $)$ returns the attractor that list must end with.

The idea is to apply $\operatorname{idAtts}\left(\varphi_{1}, \varphi_{2}\right)$ to each possible pair of SCCs $\varphi_{1} \in \operatorname{SCC}\left(S G_{g_{1}^{1}}\left(\mathcal{B N}_{1}\right)\right)$ and $\varphi_{2} \in$ $S C C\left(S G_{g_{1}^{2}}\left(\mathcal{B N}_{2}\right)\right)$. There are standard algorithms based on depth-first search for finding SCCs, such as Tarjans algorithm [25], which run in linear time $O(|V|+|E|)$ for a graph $(V, E)$. The overall performance of the proposed algorithmic approach depends on the number of SCCs and their size. Assuming that the number of combined states for the SCCs in any subnetwork is bounded by $k$ gives an upperbound of $k^{2}$ state pairs to consider. 


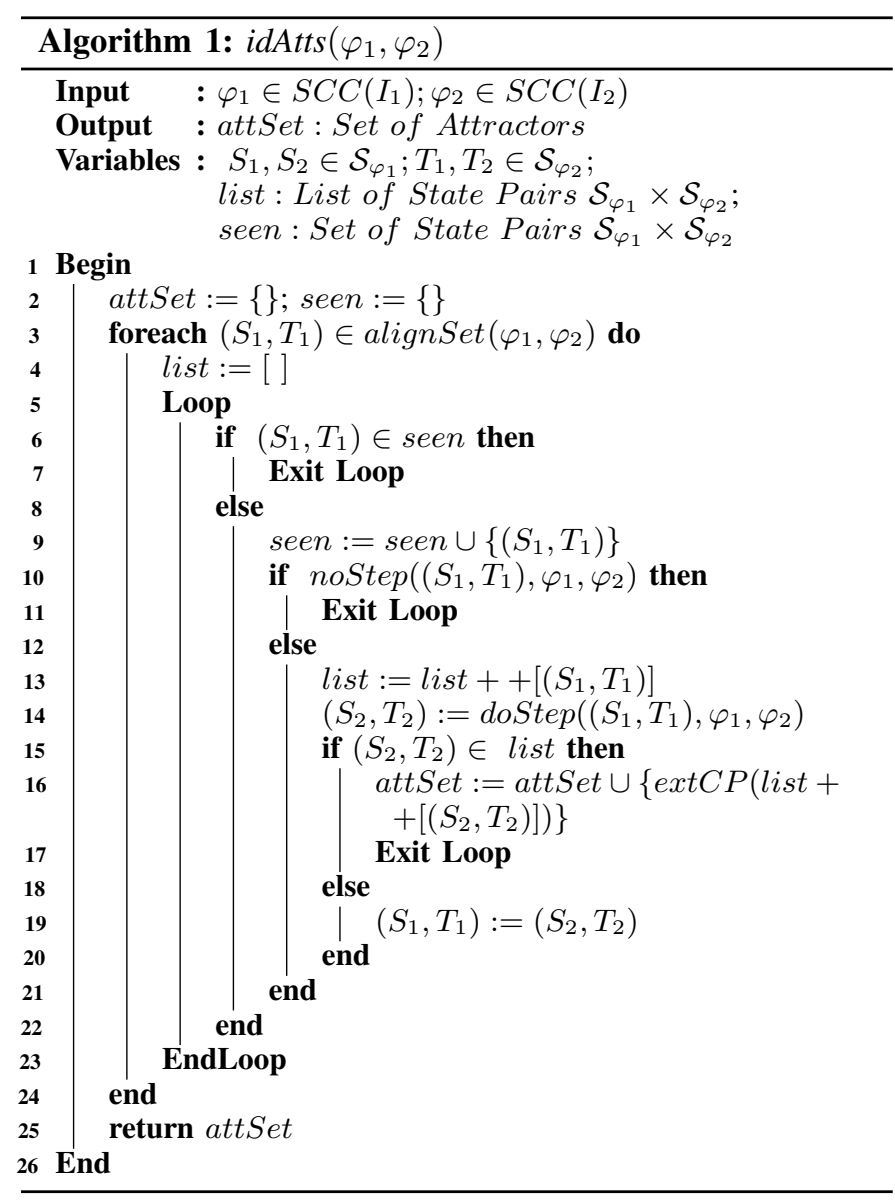

To illustrate how the algorithm works consider applying it to the composition $\mathcal{C}\left(\mathcal{B N}_{E x 1}, \mathcal{B N}_{E x 2}, g_{1}^{1}, g_{1}^{2}\right)$ (Figure 2). In this example we have two SCCs $\varphi_{1}^{1}$ and $\varphi_{2}^{1}$ for $\mathcal{B N}_{1}$, and one SCC $\varphi_{1}^{2}$ for $\mathcal{B N}_{2}$ (see Figure 4). We therefore have two possible pairs of SCCs $\left(\varphi_{1}^{1}, \varphi_{1}^{2}\right)$ and $\left(\varphi_{2}^{1}, \varphi_{1}^{2}\right)$ to apply idAtts to.

$\operatorname{idAtts}\left(\varphi_{1}^{1}, \varphi_{1}^{2}\right)$ : We have $\left|\left(\mathcal{S}_{\varphi_{1}^{1}} \times \mathcal{S}_{\varphi_{1}^{2}}\right)\right|=2$ possible state pairs but there is only one state pair $(001,00)$ that align. So idAtts will process $(001,00)$ resulting in list $=[(001,00),(001,00)]$ and $\operatorname{ext} C P($ list $)$ returns the point attractor [0010, 0010].

$\operatorname{idAtts}\left(\varphi_{2}^{1}, \varphi_{1}^{2}\right):$ We have $\left|\left(\mathcal{S}_{\varphi_{2}^{1}} \times \mathcal{S}_{\varphi_{1}^{2}}\right)\right|=6$ possible state pairs but there are only three state pairs that align

$$
\operatorname{alignSet}\left(\varphi_{2}^{1}, \varphi_{1}^{2}\right)=\{(011,00),(000,00),(101,10)\}
$$

If idAtts processes $(011,00)$ first then the result is

$$
\text { list }=[(011,00),(000,00),(101,10),(011,00)]
$$

and $\operatorname{extCP}($ list $)$ will then return the attractor $[0110,0000,1010,0110]$. Note the remaining two aligned state pairs $(000,00)$ and $(101,10)$ have already been considered in the process above.

It can be seen that the algorithm has correctly identified the attractors for $\mathcal{C}\left(\mathcal{B N}_{E x 1}, \mathcal{B N}_{E x 2}, g_{1}^{1}, g_{1}^{2}\right)$.

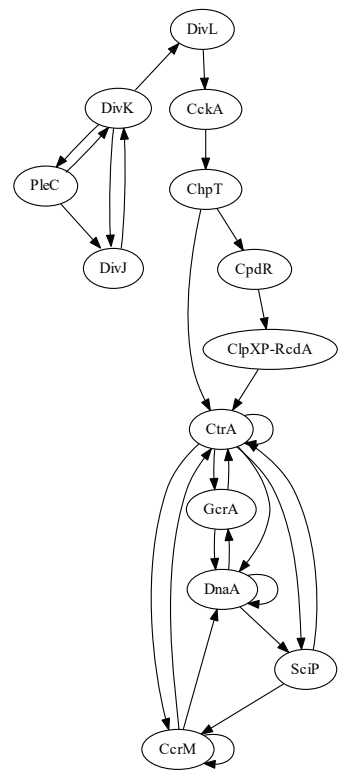

$$
\begin{aligned}
& \operatorname{Ctr} A=((C \operatorname{tr} A \vee G \operatorname{cr} A) \wedge \\
& \neg \operatorname{Ccr} M \wedge \neg S c i P) \wedge \\
& (\mathrm{ChpT} \wedge \\
& \neg C \operatorname{lp} X P-\operatorname{Rcd} A) \\
& \operatorname{Gcr} A=\operatorname{Dna} A \wedge \neg \operatorname{Ctr} A \\
& \operatorname{Dna} A=C \operatorname{tr} A \wedge C \operatorname{Cr} M \wedge \\
& \neg \operatorname{Gcr} A \wedge \neg \text { DnaA } \\
& \operatorname{Ccr} M=\operatorname{Ctr} A \wedge \neg \operatorname{Ccr} M \wedge \\
& \neg \operatorname{SciP} \\
& \text { SciP }=\operatorname{Ctr} A \wedge \neg D n a A \\
& \text { DivK }=\text { DivJ } \wedge \neg P l e C \\
& \text { PleC }=\neg D i v K \\
& \text { DivJ }=\text { DivK } \wedge \neg P l e C \\
& \text { DivL }=\neg D i v K \\
& \text { CckA }=\text { DivL } \\
& \text { ChpT }=\text { CckA } \\
& C p d R=C h p T \\
& C l p X P-\operatorname{Rcd} A=\neg C p d R
\end{aligned}
$$

Fig. 5: The Boolean network $\mathcal{B N}_{C c}$ for the regulatory network for cell differentiation in C. crescentus (based on [21], [23])

The algorithm idAtts has been used as the basis for developing a prototype support tool ${ }^{1}$ for compositionally identifying attractors. The tool is implemented in Python using the NetworkX package [6] which has tools to represent and manipulate network structures. The tool reads in state graphs for the subnetworks using and then generates the required interference state graphs. It then uses an implementation of Tarjans algorithm [19], [25] to identify the required SCCs. It then applies an implementation of the idAtts algorithm to the possible pairs of SCCs and returns all the attractors identified.

\section{A Case Study}

In this section, we illustrate the practical application of our techniques and tools with a case study based on analysing a Boolean network for the regulatory network for cell differentiation in the bacteria Caulobacter crescentus [21], [23].

\section{A. Qualitative Model for Cell Differentiation}

The bacteria $C$. crescentus [13] is a model organism for studying cellular differentiation and asymmetric division in bacteria as its division cycle generates two phenotypes: the stalked and the swarmer cell types. A set of Boolean network models for analysing the regulatory network behind cell differentiation in C. crescentus were proposed in [21], [23] and we use these as the basis for the Boolean network $\mathcal{B N}_{C c}$ presented in Figure 5.

Based on the approach suggested in [21], [23] we decompose this Boolean network into two subnetworks $\mathcal{B N}_{C c 1}$ and $\mathcal{B N}_{C c 2}$ (see Figure 6) by splitting the entity $C \operatorname{tr} A$ into

\footnotetext{
${ }^{1}$ For more information about the tool and to obtain a copy, please email the authors.
} 
two entities $C \operatorname{tr} A_{a}$ and $C \operatorname{tr} A_{b}$ with corresponding next-state functions

$$
\begin{gathered}
C \operatorname{tr} A_{a}=((C \operatorname{tr} A \vee G \operatorname{cr} A) \wedge \neg C \operatorname{cr} M \wedge \neg S c i P) \\
C \operatorname{tr} A_{b}=(C h p T \wedge \neg C \operatorname{lp} X P-\operatorname{Rcd} A)
\end{gathered}
$$

It can be shown that the composition $\mathcal{C}\left(\mathcal{B N}_{C c 1}, \mathcal{B N}_{C c 2}, C \operatorname{tr} A_{a}, C \operatorname{tr} A_{b}\right)$ of the two subnetworks on the entities $\operatorname{Ctr} A_{a}$ and $C \operatorname{tr} A_{b}$ using conjunction does correctly result in the original Boolean network $\mathcal{B N}_{C c}$.

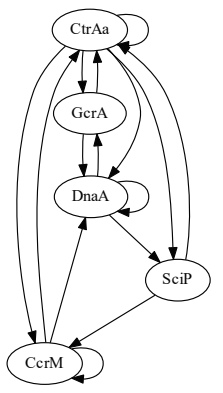

$\mathcal{B N}_{C c 1}$

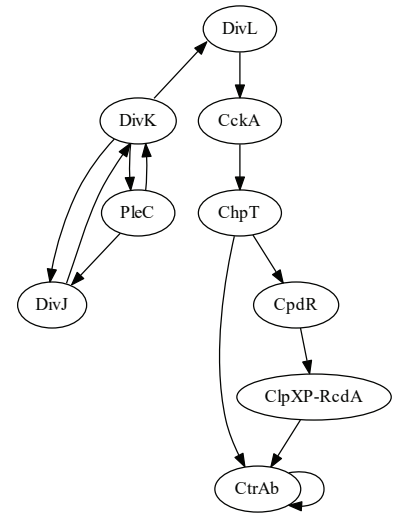

$\mathcal{B N}_{C c 2}$
Fig. 6: Subnetworks $\mathcal{B N}_{C c 1}$ and $\mathcal{B N}_{C c 2}$ derived for $\mathcal{B N}_{C c}$ (based on [21], [23]), where entity $C \operatorname{tr} A$ is split into $C \operatorname{tr} A_{a}$ in $\mathcal{B N}_{C c 1}$ and $C \operatorname{tr} A_{b}$ in $\mathcal{B N}_{C c 2}$

This decomposition can be seen to dramatically reduces the state space that needs to be considered; the original model had $2^{13}=8192$ global states while the two submodels have $2^{5}=32$ and $2^{9}=512$.

\section{B. Application of our Approach}

We now consider applying our techniques and tool to $\mathcal{B N}_{C c 1}$ and $\mathcal{B N}_{C c 2}$ to identify the attractors of the composed model $\mathcal{B N}_{C c}$. We note that the purpose of this work is not to produce any new biological insight but to provide a clear example of the practical application of the developed techniques and tools.

The process starts by generating the state graphs for $\mathcal{B N}_{C c 1}$ and $\mathcal{B N}_{C c 2}$ using a standard tool such as GinSim [18] or BoolNet [17]. These are then usd by our tool to generate the corresponding interference state graphs. The support tool then identifies the SCCs in the interference state graphs: there are two SCCs $\varphi_{1}^{1}$ and $\varphi_{2}^{1}$ in $S G_{C t r A_{a}}\left(\mathcal{B N}_{C c 1}\right)$; and two SCCs $\varphi_{1}^{2}$ and $\varphi_{2}^{2}$ in $S G_{C t r A_{b}}\left(\mathcal{B N} \mathcal{N}_{C c 2}\right)$ (see Figure 7). The total number of possible state pairs is 15 but the tool only considers the eight state pairs that align (see below).

We are then able to apply the implementation of idAtts to the four possible SCC pairs. The result is that the support tool correctly identifies the three attractors for $\mathcal{B N}_{C c}$ (this was verified using existing tools such as BoolNet [17]).

The following summarises the results produced by the tool:

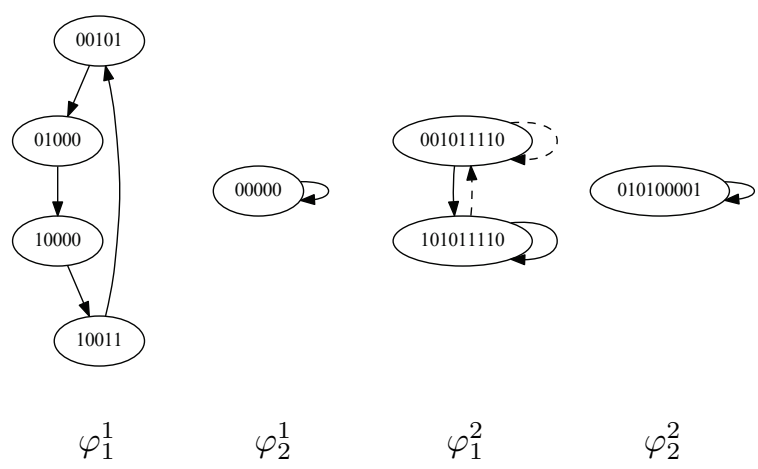

Fig. 7: The identified SCCs $\varphi_{1}^{1}$ and $\varphi_{2}^{1}$ for $\left.S G_{C t r A_{a}}\left(\mathcal{B N}_{C c 1}\right)\right)$ (state order is $\operatorname{Ctr} A_{a}, \operatorname{Gcr} A, \operatorname{Dna} A, \operatorname{Cr} M, \operatorname{SciP}$ ), and $\varphi_{1}^{2}$ and $\varphi_{2}^{2}$ for $S G_{C t r A_{b}}\left(\mathcal{B N}_{C c 2}\right)$ ) (state order is $C \operatorname{tr} A_{b}, \operatorname{DivK}$, PleC, DivJ, DivL, CckA, ChpT, CpdR, ClpXP-RcdA)

1) $\operatorname{idAtts}\left(\varphi_{1}^{1}, \varphi_{1}^{2}\right)$ : There are four aligned state pairs to consider. Starting with the state pair $(00101,001011110)$ the result is the single list of state pairs $\{[(00101,001011110),(01000,001011110),(10000,101011110)$, (10011, 10101110), (00101, 001011110)]\} which are merged to produce the attractor [0010101011110, 0100001011110, 1000001011110, 1001101011110, 0010101011110].

The other aligned state pairs $\{(01000,001011110)$, $(10000,101011110),(10011,10101110)\}$ would produce the same attractor but are not processed as they have already been added to the seen list.

2) $\operatorname{idAtts}\left(\varphi_{1}^{1}, \varphi_{2}^{2}\right)$ : There are two aligned state pairs to consider. The algorithm returns \{\} and no attractors are found.

3) $\operatorname{idAtts}\left(\varphi_{2}^{1}, \varphi_{1}^{2}\right)$ : There is one aligned state pair $(00000,001011110)$ that results in the single list of state pairs $\{[(00000,001011110),(00000,001011110)]\}$

which are merged to produce the attractor

[0000001011110, 0000001011110].

4) $\operatorname{idAtts}\left(\varphi_{2}^{1}, \varphi_{2}^{2}\right)$ : There is one aligned state pair $(00000,010100001)$ that results in the single list of state pairs $\{[(00000,010100001),(00000,010100001)]\}$

which are merged to produce the attractor

[0000010100001, 0000010100001].

\section{CONCLUSIONS}

This paper presented initial results on extending an existing compositional framework [3], [4] for Boolean networks with new novel techniques and tools for compositionally identifying attractors. This work was important as providing support for compositional attractor analysis is crucial for addressing the state space explosion problem and for ensuring the practical applicability of the compositional framework for engineering 
and analysing Boolean networks. While a range of work on compositionally identifying attractors already exists in the literature (for example, see [5], [9], [16], [28]-[30]) the approach here is novel as the techniques are incorporated into a framework for compositionally constructing models which supports engineering biological systems.

The new results we present provide the foundation for a wide range of further work. For example, the current initial approach focused on composing two Boolean networks but this is too restrictive in practice. Work is now underway to generalise the framework to allow the arbitrary compositions of multiple models and to extend the attractor identification techniques to this new general setting.

The performance of the new techniques also needs further investigation. We plan to develop an enhanced version of the tool support for our techniques and to evaluate this using a range of experimental studies based on comparing our approach to other existing tools.

The original compositional framework was developed to support the engineering of Boolean networks from basic parts in order to help address current challenges in synthetic biology. However, it is also interesting to consider using it as a basis for analysing existing Boolean networks (as was done in the case study in Section 5). To facilitate this we intend to consider developing techniques for automatically decomposing a large Boolean network into parts to aid their analysis and the identification of key subnetworks.

\section{ACKNOWLEDGMENTS}

We would like to thank Hanadi Alkhudhayr for many interesting discussions on Boolean network composition. We also like to thank the anonymous referees for their helpful comments.

\section{REFERENCES}

[1] Tatsuya Akutsu, Satoru Miyano, Satoru Kuhara, et al. Identification of genetic networks from a small number of gene expression patterns under the boolean network model. Pacific Symposium on Biocomputing, 4:17-28, 1999.

[2] H. Alkhudhayr. Developing a Compositional Framework for the Construction and Analysis of Boolean Networks. PhD thesis, School of Computing, Newcastle University, 2020.

[3] H. Alkhudhayr and J. Steggles. A compositional framework for boolean networks. Biosystems, 186:103960, 2019.

[4] Hanadi Alkhudhayr and Jason Steggles. A formal framework for composing qualitative models of biological systems. In C Martin-Vide, $\mathrm{R}$ Neruda, and M Vega-Rodriguez, editors, Theory and Practice of Natural Computing, TPNC2017, LNCS 10687, pages 25-36. Springer, 2017.

[5] Wensheng Guo, Guowu Yang, Wei Wu, Lei He, and Mingyu Sun. A parallel attractor finding algorithm based on boolean satisfiability for genetic regulatory networks. PloS one, 9(4):e94258, 2014.

[6] Aric Hagberg, Pieter Swart, and Daniel S Chult. Exploring network structure, dynamics, and function using networkx. Technical report, Los Alamos National Lab.(LANL), Los Alamos, NM (United States), 2008.

[7] Inman Harvey and Terry Bossomaier. Time out of joint: Attractors in asynchronous random boolean networks. In Proceedings of the Fourth European Conference on Artificial Life, pages 67-75. MIT Press, Cambridge, 1997.

[8] Tomáš Helikar, John Konvalina, Jack Heidel, and Jim A. Rogers. Emergent decision-making in biological signal transduction networks. Proceedings of the National Academy of Sciences, 105(6):1913-1918, 2008.
[9] Changki Hong, Jeewon Hwang, Kwang-Hyun Cho, and Insik Shin. An efficient steady-state analysis method for large boolean networks with high maximum node connectivity. PLOS ONE, 10(12):e0145734, 2015.

[10] Sui Huang and Donald E Ingber. Shape-dependent control of cell growth, differentiation, and apoptosis: switching between attractors in cell regulatory networks. Experimental cell research, 261(1):91-103, 2000.

[11] Stuart A. Kauffman. Metabolic stability and epigenesis in randomly constructed genetic nets. Journal of Theoretical Biology, 22(3):437467, 1969 .

[12] Stuart A. Kauffman. The origins of order: Self organization and selection in evolution. Oxford University Press, USA, 1993.

[13] Michael T Laub, Lucy Shapiro, and Harley H McAdams. Systems biology of caulobacter. Annu. Rev. Genet., 41:429-441, 2007.

[14] F Li, T Long, Y Lu, Q Ouyang, and C Tang. The yeast cell-cycle network is robustly designed. Proceedings of the National Academy of Sciences, 101(14):47814786, 2004.

[15] B. W. Miller and D. L. Ranum. Problem Solving with Algorithms and Data Structures Using Python. Franklin, Beedle and Associates Inc, 2 edition, 2011.

[16] Andrzej Mizera, Jun Pang, Hongyang Qu, and Qixia Yuan. A new decomposition method for attractor detection in large synchronous boolean networks. In International Symposium on Dependable Software Engineering: Theories, Tools, and Applications. SETTA 2017, volume LNCS 10606, pages 232-249. Springer, 2017.

[17] Christoph Müssel, Martin Hopfensitz, and Hans A Kestler. Boolnetan r package for generation, reconstruction and analysis of boolean networks. Bioinformatics, 26(10):1378-1380, 2010.

[18] Aurélien Naldi, Céline Hernandez, Wassim Abou-Jaoudé, Pedro T Monteiro, Claudine Chaouiya, and Denis Thieffry. Logical modeling and analysis of cellular regulatory networks with ginsim 3.0. Frontiers in physiology, 9:646, 2018.

[19] Esko Nuutila and Eljas Soisalon-Soininen. On finding the strongly connected components in a directed graph. Information processing letters, 49(1):9-14, 1994.

[20] Sona Pandey, Rui-Sheng Wang, Liza Wilson, Song Li, Zhixin Zhao, Timothy Gookin, Sarah Assmann, and Reka Albert. Boolean modeling of transcriptome data reveals novel modes of heterotrimeric g-protein action. Molecular Systems Biology, 6(1):2375-2387, 2010.

[21] César Quiñones-Valles, Ismael Sánchez-Osorio, and Agustino MartínezAntonio. Dynamical modeling of the cell cycle and cell fate emergence in caulobacter crescentus. PloS one, 9(11):e111116, 2014.

[22] Assieh Saadatpour, Rui-Sheng Wang, Aijun Liao, Xin Liu, Thomas P Loughran, Istvn Albert, and Rka Albert. Dynamical and structural analysis of a t cell survival network identifies novel candidate therapeutic targets for large granular lymphocyte leukemia. PLOS Computational Biology, 7(11):e1002267, 2011.

[23] Ismael Sánchez-Osorio, Carlos A Hernández-Martínez, and Agustino Martínez-Antonio. Modeling asymmetric cell division in caulobacter crescentus using a boolean logic approach. In Asymmetric Cell Division in Development, Differentiation and Cancer, pages 1-21. Springer, 2017.

[24] Julian D. Schwab, Silke D. Khlwein, Nensi Ikonomi, Michael Khl, and Hans A. Kestler. Concepts in boolean network modeling: What do they all mean? Computational and Structural Biotechnology Journal, 18:571582, 2020.

[25] Robert Tarjan. Depth-first search and linear graph algorithms. SIAM journal on computing, 1(2):146-160, 1972.

[26] Denis Thieffry and René Thomas. Dynamical behaviour of biological regulatory networks-II. Immunity control in bacteriophage lambda. Bulletin of Mathematical Biology, 57(2):277-297, 1995.

[27] Andrew Wuensche. Aggregation algorithm towards large-scale boolean network analysis. In G. Schlosser and G. P. Wagner, editors, Modularity in Development and Evolution, chapter 13, pages 288-311. Uni Of Chicago Press, 2004.

[28] Qixia Yuan, Andrzej Mizera, Jun Pang, and Hongyang Qu. A new decomposition-based method for detecting attractors in synchronous boolean networks. Science of Computer Programming, 180:18-35, 2019.

[29] Qixia Yuan, Hongyang Qu, Jun Pang, and Andrzej Mizera. Improving bdd-based attractor detection for synchronous boolean networks. Science China Information Sciences, 59(8):1-16, 2016.

[30] Yin Zhao, Jongrae Kim, and Maurizio Filippone. Aggregation algorithm towards large-scale boolean network analysis. IEEE Transactions on Automatic Control, 58(8):1976-1985, 2013. 